\title{
A Review - Water Quality Monitoring System
}

\section{R. J. Sapkal ${ }^{1}$, Pooja Wattamwar ${ }^{2}$, Rani Waghmode ${ }^{3}$, Umrunnisa Tamboli $^{4}$}

${ }^{1}$ Professor, Department of Electronics and Telecommunications Engineering, BVCOEW, Pune, Mahashtra, India

2,3,4BE, Department of Electronics and Telecommunications Engineering, BVCOEW, Pune, Mahashtra, India

\begin{abstract}
This paper describes the main reason for need of effective and efficient water level monitoring and control of water quality in flat system tends to keeping the human resources healthy and sustainable, and to reduce the usage of water for household purposes. Due to climate changes and variability so many huge impacts are caused by the water system to the natural environment. Incredible methods are used by collecting water samples, testing and analyses in water laboratories alone. However, It is not always easy to be captured, analyses and fast dissemination of information to relevant users for making timely and well-versed decisions. In this project Water Sensor System prototype is developed for water level and quality monitoring in society is presented. These kind of growth was introduced by the assessment of widespread atmosphere that Including accessibility of cellular network Coverage at the site of process.
\end{abstract}

Keywords : IOT Module, Level Sensor, Water Quality Mobile Phone Platforms, Sensor Nodes.

\section{INTRODUCTION}

The importance of maintaining good water quality is to increasing the need for advance technology to help monitor the water condition and maintain the water condition. However ,In this paper, we are using the lake water for monitoring that provides some important service such as water for drinking, domestic purpose ,sites for recreational activity ,and important fisheries and also agricultural purpose .If pollution are involved in lake water ,then it can reduce the quality of water and so that it is unsafe to drink as well as harm organism that lives in water. The negative collisions of climate change and inconsistency are compounding the accessible challenges. As a result, increasing number of human activities has progressively speed-up contagion and damage to fresh water resources in the lakes. This traditional advance of water quality monitoring based on variety and subsequent analyses in water laboratories is exclusive, time- consuming and has a low promise both in time and space. In this paper, a planned IOT based water quality monitoring system sample is presented. The system consists of a ARM7 microcontroller, a set of water quality sensors, and a network connection module. It measures water quality parameters including Turbidity, electrical conductivity (EC) values in immediate. The experimental results showed that the system has great prospect and can be used to operate in real world environment for optimum control of aquaculture environment. Further more, the prototype is suitable for long-term outdoor environments and implement low-cost gateway module. The Smart Water Quality Monitoring System will measure the following water parameters for analysis; Conductivity and level monitoring. While monitoring these parameters, it is perceived 
that one should receive a stable set of results. Therefore a continuous series of anomalous measurements would indicate the potential introduction of a water pollutant and the user will be notified of this activity with the aid of IOT technology. False positives, such as anomalous readings over a short period of time, will be recorded but not treated as an alert. Hence, with the successful implementation of this monitoring approach.

\section{LITRATURE SURVEY}

\section{IOT Based Water Quality Monitoring}

This paper investigates a real-time water quality monitoring system by using a proposed broker less publisher subscriber (pub/sub) architecture framework. On the system, sensors sense the water measurement metrics, including temperature, $\mathrm{pH}$, and dissolved oxygen level. All collected data are stored in a database and computed stochastically for further analysis on water quality. A complementary experiment compares the proposed pub/sub architecture and MQTT, a lightweight protocol on which IoT mostly uses, to show better performance of the proposed architecture in case of network latency and throughput for diverse message payload size, thus suggesting the future IoT implementation of the system.

Author: Alif Akbar Pranata, Jae Min Lee

Publication \& year: IEEE 2017

\section{Water Quality Monitoring Using Interner of Things}

The paper suggests an Internet of Things (IoT) based system implementation by embedding the Radio Frequency Identification (RFID) system, Wireless Sensor Network (WSN) platform and Internet Protocol (IP) based communication into a single platform for water quality monitoring (WQM) purpose. The suggested radio frequency for the proposed WSN communication to be deployed in vegetation area is $920 \mathrm{MHz}$. The measured water parameter in this proposed system is $\mathrm{pH}$ level by using an analog $\mathrm{pH}$ sensor.

Author: Hafiz Kamaludin, Widad Ismail

Publication \& year: IEEE 2017

\section{Water Quality Monitoring and Control}

The main reason for need of effective and efficient monitoring and control of water quality in lakes, rivers and sea tends to keeping the human resources healthy and sustainable, and to increase population growth and urbanization. Due to climate changes and variability so many huge impacts are caused by the water system to the natural environment. Incredible methods are used by collecting water samples, testing and analyses in water laboratories alone. In this paper, Water Sensor Network (WSN) system prototype is developed for water quality monitoring in Lakes is presented. These kind of growth was introduced by the assessment of widespread atmosphere that including accessibility of cellular network coverage at the site of process.

Author: Karuppasamy M.E., Ph. D, B Abinaya

Publication \& year: IRJET 2017

Figure and Table

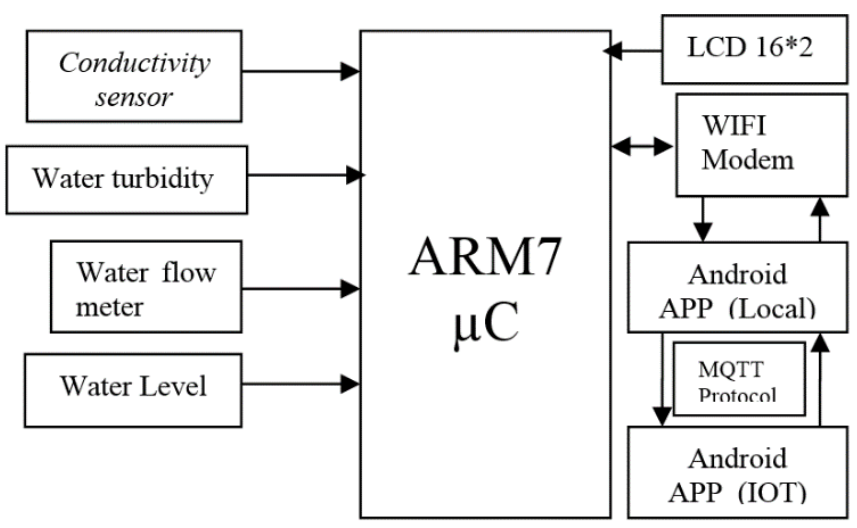

\section{Block Diagram Explanation}

In today's world the water monitoring is of major concern. In majority of cases the water which is supplied is contaminated. For this a robust method 
for water quality monitoring is required. Keeping this in mind we have designed a Embedded hardware which will monitor the quality of water and display the results on android APP. In our project we have considered the scenario of Society based water tank which is situated below the ground. The water is then pumped into the overhead water head tank for use of people. Here the $\mu \mathrm{C}$ will continuously monitor the water quality by measuring the water turbidity to check for impurities suspended in water and conductivity sensor to check content of water. If both the parameters are within set limits then the water is certified to for drinking. Here we are also designing a system for water management. The watchman will have a local APP which is connected to embedded hardware via WIFI. The user can request to turn on Motor. The $\mu \mathrm{c}$ will check whether water in the base tank is more than set point. If the water is less then motor is stopped. Also the water allotted to the society tank is limited. We have interfaced a water flow sensor. The $\mu \mathrm{C}$ will continuously sense the water quantity released. If the water released is more than set point then water is stopped. In this way we are managing the water distributed to societies using water flow meter. Here if the water is purity is high the $\mu \mathrm{C}$ will send all the sensor data to APP over WIFI. The APP will compare the sensor readings with internal set points and display the output to be fit for drinking.

The Local APP will send all the data to IOT based APP once in a day to log the water quality and water quantity released every day. Local app is used to develop to send the data to the end user. MQTT is a machine-to-machine (M2M)"Internet of Things" connectivity protocol. In the proposed system water quality parameters are measured by different sensors. So in order to meet all these requirements and technologies is used such as MQTT which allows publishing and subscribing of data between the sensors and end device. And with the help of MQTT algorithm there will be simultaneous flow of data between sensors and the server.

\section{Algorithm}

1. Start

2. Init LCD

3. Display project name

4. "A"

5. Select channel 1

6. Read ADC

7. Store and display Turbidity on LCD

8. IS Turbidity $>=$ High? $\mathrm{N} \rightarrow " \mathrm{~B} "$

9. $\mathrm{Y} \rightarrow$ Send Indication to IOT server

10. "B"

11. Select channel 2

12. Read ADC

13. Store and display conductivity value on LCD

14. IS Conductivity $>=85 \%$ or Conductivity $<=15 \%$ ? $\mathrm{N} \rightarrow$ "C"

15. $\mathrm{Y} \rightarrow$ Send Indication to IOT server

16. "C"

17. Select channel 3

18. Read ADC

19. Store and display Water Level on LCD

20. IS WL $<=20 \%$ ? $N \rightarrow " D "$

21. $\mathrm{Y} \rightarrow$ Send Indication to IOT server

22. "D"

23. Count Pulses

24. Store and display Water flow on LCD

25. Is Send Motor ON Button ON Pressed? N $\rightarrow$ "A"

26. Is Water Level $>20 \%$ \& \& Conductivity $=$ normal? $\mathrm{N} \rightarrow$ Motor OFF $\rightarrow " \mathrm{~A} "$

27. $\mathrm{Y} \rightarrow$ Motor $\mathrm{ON} \rightarrow " \mathrm{~A} "$ 


\section{Simulation Circuit Layout}

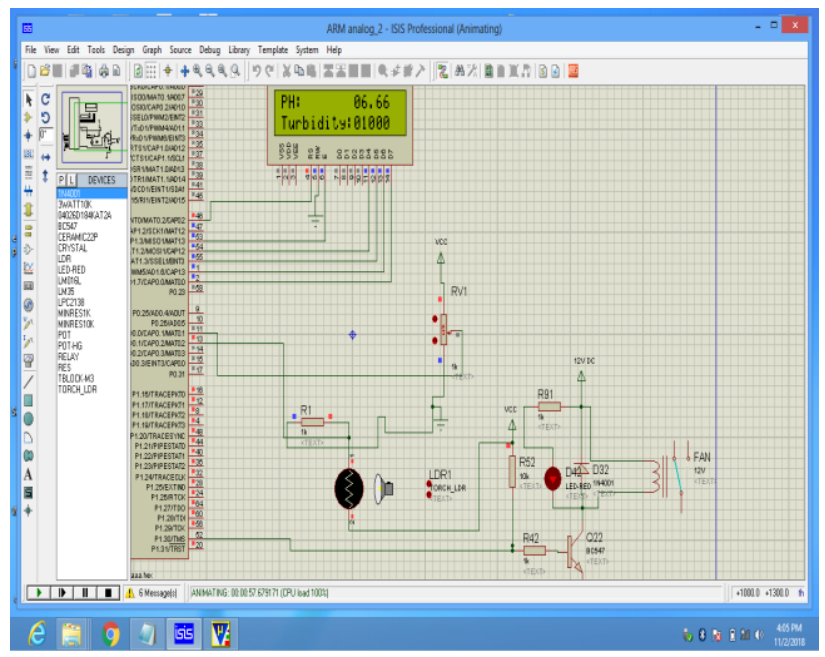

\section{Protocol Used (MQTT protocol)}

MQTT is a machine-to-machine (M2M)/"Internet of Things" connectivity protocol. It is an extremely lightweight publish/subscribe messaging transport. It is useful for connections with remote locations where a small code footprint is required and/or network bandwidth is at a premium. MQTT is a publish/subscribe protocol that allows edge-ofnetwork devices to publish to a broker. Clients connect to this broker, which then mediates communication between the two devices. Each device can subscribe, or register, to particular topics. In the proposed system water quality parameters are measured by different sensors. So in order to meet all these requirements and technologies is used such as MQTT which allows publishing and subscribing of data between the sensors and end device. And with the help of MQTT algorithm there will be simultaneous flow of data between sensors and the server. The main purpose of using IOT approach to monitor water quality using MQTT algorithm is to develop a system which provides end user a useful data used. Conventionally, the water sample are collected from different places and tested rigorously by scientist in the laboratory using many techniques to determine the water quality. Therefore older method where time consuming process but now IOT has the potential to modernize the water production, as more and more of its technology is connected to the web. This IOT approach is far better than conventional methods since it is cost friendly, faster and easy to use. Security is an essential factor for any system security at both the device and network level is critical to the operation of IOT.

\section{Android APP}

The Water Quality mobile app is a water-monitoring data-collection and learning tool designed for use by educators and their students, citizen scientists, and researchers. The app helps users log and share data they collect in the field, interpret the data through learning pop-ups, and export it to create databases. Available for an Android (phones and tablets) mobile devices, this app includes data entry, identification, and learning features about chemical (e.g. turbidity), physical (e.g. water temperature, turbidity) waterquality parameters. Mobile phone applications are discussed, together with advantages and disadvantages of different technologies.

\section{Specifications}

a) Project Specifications:

- Project board: $10 \mathrm{~cm} \mathrm{x} 10 \mathrm{~cm}$

- Project Enclosure: 1feet $\mathrm{x} 1$ feet

- Project supply: 230V, 1 Ampere

- Power specifications: $12 \mathrm{~V}$ x $500 \mathrm{ma}=6 \mathrm{Watt}$

b) Hardware Specifications:

Microcontroller: LPC2138 ARM7 Microcontroller, 32K RAM \& ROM, 40 I/O, 2 serial Ports

- Turbidity sensor: LED+LDR

- PH: Electrode sensor

- Water level: Float sensor

- Water flow meter: Rotating pulse output meter 
- Display: $\quad$ LCD16*2, $\quad$ Voltage $\rightarrow 5 \mathrm{~V}$, Current $\rightarrow 10 \mathrm{ma}$

- WIFI Module: ESP8266 module, 30meters range, $5 \mathrm{~V}, 50 \mathrm{ma}$

- Relay: 230V,5A

- Buzzer: 5V, 10ma

c) Software Requirements:

- Compiler: KEIL3

- Programming Language: Embedded C

- Programming Platform: Flash Magic

- Android APP: Basic for Android Software

\section{ACKNOWLEDGMENT}

We would like to thank our Principal And Head of the Department Dr. S.R. Patil, our guide Prof. R.J. Sapkal for their valuable advice and technical assistance.

\section{REFERENCES}

[1]. 'International decade for action 'water for life' 2005-2015."

[Online]Available:http://www.un.org/waterforl ifedecade/sanitation.shtml

[2]. C. H. Tseng, "Coordinator traffic diffusion for data-intensive zigbee transmission in real-time electrocardiography monitoring," IEEE Transactions on Biomedical Engineering, vol. 60, no. 12, pp. 3340-3346,Dec. 2013.

[3]. S. K. Chen, T. Kao, C. T. Chan, C. N. Huang, C. Y. Chiang, C. Y. Lai, T. H. Tung, and P. C. Wang, "A reliable transmission protocol for zigbee based wireless patient monitoring," IEEE Transactions on Information Technology in Biomedicine, vol. 16, no. 1, pp. 6-16, Jan. 2012.

[4]. R. Manasrah, M. Raheed, and M. I. Badran,"Relationships between water temperature, nutrients and dissolved oxygen in the northern gulf of a qaba, red sea,"
OCEANOLOGIA, vol. 48, no. 2, pp. 237-253, Apr.2006.

[5]. J. Ashton and L. Geary, "The effects of temperature on $\mathrm{PH}$ measurement," Reagecon Delivering the Correct Result, Shannon, Co.Clara, Ireland, Apr. 2011.

[6]. N. A. Cloete, R. Malekian, and L. Nair, "Design of smart sensors for real-time water quality monitoring," IEEE Access, vol. 4, pp. 39753990,2016.

[7]. Design spark,'11 internet of things (IOT) protocol you need to know about, accessed December 10, 2016.

[8]. $\mathrm{N}$ dijaykumar, $\mathrm{R}$ Ramya, "The real time monitoring of water quality in IOT environment" International conference on circuit, power and computing technologies IEEE, 2015.

\section{BIOGRAPHIES}

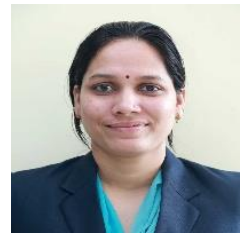

Prof. R.J. Sapkal, Professor at Bharti Vidyapeet's Collage Of Engineering for Women, Katraj. She has Published Six Papers in International Journal and one at IEEE conference.

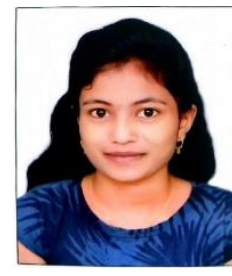

Pooja Wattamwar receiving the B.E. degree from Pune University, India. She is currently a final year B.E. student of Bharti Vidyapeet's Collage Of Engineering for Women, Katraj.

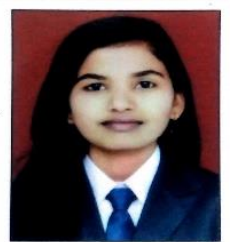

Rani Waghmode receiving the B.E. degree from Pune University, India. She is currently a final year B.E. student of Bharti Vidyapeet's Collage Of Engineering for Women, Katraj. 


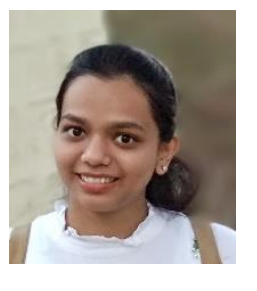

Umrunnissa Tamboli receiving the B.E. degree from Pune University, India. She is currently a final year B.E. student of Bharti Vidyapeet's Collage Of Engineering for Women, Katraj.

\section{Cite this article as :}

R. J. Sapkal, Pooja Wattamwar, Rani Waghmode, Umrunnisa Tamboli, "A Review - Water Quality Monitoring System", International Journal of Scientific Research in Science and Technology (IJSRST), Online ISSN : 2395-602X, Print ISSN : 2395-6011, Volume 6 Issue 2, pp. 76-81, March-April 2019. Available at doi : https://doi.org/10.32628/IJSRST19626 Journal URL : http://ijsrst.com/IJSRST19626 